\title{
The role of $h L H X 6$-HMR as a methylation biomarker for early diagnosis of cervical cancer
}

\author{
SAMIL JUNG ${ }^{1 *}$, DONGJUN JEONG ${ }^{2 *}$, JINSUN KIM ${ }^{1}$, LISHA YI ${ }^{1}$, KEUNHOE KOO ${ }^{1}$, \\ JAEHYOUK LEE ${ }^{1}$, SOON-DUCK LEE ${ }^{1}$, JIN-WHA PARK ${ }^{1}$, BOOGI CHANG ${ }^{1}$, \\ CHANG-HWAN KIM ${ }^{2}$, CHANG-JIN KIM ${ }^{2}$ and MYEONG-SOK LEE ${ }^{1}$ \\ ${ }^{1}$ Division of Biological Science and Research Center for Women's Diseases, Sookmyung Women's University, \\ Seoul 140-742; ${ }^{2}$ Department of Pathology, College of Medicine, Soonchunhyang University \\ 366-1, Ssangyong-Dong, Chonan 330-090, Korea
}

Received December 23, 2009; Accepted February 2, 2010

DOI: 10.3892/or_00000811

\begin{abstract}
The homo sapiens LIM homeobox domain LHX6 gene, $h L H X 6$, is a putative transcription regulator with homeodomain. Multiple cytosine guanine dinucleotides ( $\mathrm{CpG}$ island) are found in the genomic sequences between exon $4 \mathrm{a}$ and exon 5 of the gene encoding hLHX6s (alternative short isoform of $h L H X 6$ gene). This specific $\mathrm{CpG}$ island, $h L H X 6$ $\mathrm{HMR}$, is found frequently hypermethylated in 7 cervical cancer cell lines as shown in MSP, BSP, and COBRA assays. Methylation densities were also investigated with human tissue samples with a distinctive degree of malignant transformation. Our data showed that the $h L H X 6$-HMR was rarely or partly methylated in the normal and CIN I cells, respectively. In contrast, it was frequently hypermethylated in CIN II, CIN III, and invasive carcinoma cells. In summary, this methylation study led to two conclusions. First, hLHX6HMR hypermethylation is exclusively associated with cervical carcinogenesis. Second, the epigenetic change in hLHX6HMR seems to start at CIN I, relatively early stage of cervical cancer development. Therefore, $h L H X 6$-HMR can be used as an effective and sensitive methylation biomarker for early diagnosis of cervical cancer.
\end{abstract}

Correspondence to: Dr Myeong-Sok Lee, Division of Biological Science and Research Center for Women's Diseases, Sookmyung Women's University, Seoul 140-742, Korea

E-mail: mslee@sookmyung.ac.kr

Dr Chang-Jin Kim, Department of Pathology, College of Medicine, Soonchunhyang University, 366-1, Ssangyong-Dong, Chonan 330-090, Korea

E-mail: cjkim@sch.ac.kr

${ }^{*}$ Contributed equally

Key words: epigenetics, methylation biomarker, cervical cancer, hLHX6-HMR

\section{Introduction}

Cervical cancer is an malignant tumor of the cervix uteri or cervical area. In spite of various advanced treatments, cervical cancer still remains a fatal disease worldwide in women (1). When infected by human papillomavirus (HPV), the cervical epithelial cells develop from premalignant cervical lesions to malignant invasive cancer via complex multistep processes (2-5). The multistep carcinogenesis can be classified into five groups: normal, nervical intraepithelial neoplasia I (CIN I, mild dysplasia), cervical intraepithelial neoplasia II (CIN II, moderate dysplasia), cervical intraepithelial neoplasia III (CIN III, severe dysplasia), and invasive cervical carcinoma $(6,7)$. Fortunately, most cases of CINs do not lead to the development of cervical cancer and only a small percentage of cases becomes cervical cancer if they are not well treated (7). Moreover, this process is a relatively slow event with a long interval between infection and cancer. Therefore, the development of diagnostic system for early detection has been considered as one of the most powerful tools in the diagnostic area. Several such diagnostic technologies (e.g., Pap smear screening or HPV testing) have been developed (7). However, their efficiency has been questioned for many reasons including sampling, interpretation errors, cost, and low specificity and sensitivity.

Currently, many researchers are interested in developing diagnostic tools for the early detection of various cancers using epigenetic alterations in the oncogenes and tumor suppressor genes. Representative epigenetic alteration is the hypermethylation at cytosine guanine dinucleotide $(\mathrm{CpG})$ island of genomic sequence (8). DNA methylation has been considered to be the most useful tool for the detection of a variety of cancers (9). CpG island methylation is the addition of a methyl group on cytosine of $\mathrm{CpG}$ dinucleotides. Hypermethylation in the promoter region of potential tumor suppressor genes and DNA repair genes often leads to the repressed gene expression and therefore loss of normal function. This epigenetic silencing is now recognized as a frequent event in the carcinogenesis of many cancer types including cervical cancer (10-16). Methylated cytosines are absent in the gene promoter under normal physiological 
Table I. Human cervix tissue samples used in MSP and BSP analysis.

\begin{tabular}{|c|c|c|c|c|c|}
\hline \multirow[b]{2}{*}{ No. ${ }^{a}$} & \multicolumn{5}{|c|}{ Diagnosis samples ${ }^{\mathrm{b}}$ (Age) } \\
\hline & Normal $^{c}$ & CIN I & CIN II & CIN III & Carcinoma \\
\hline 1 & $08-3488-1 d(48)$ & 07-692 (44) & $07-4215(34)$ & $07-852(51)$ & $7227(75)$ \\
\hline 2 & 08-7568-1b (49) & $07-949(32)$ & $07-4406(27)$ & $07-1631(48)$ & $12593(42)$ \\
\hline 3 & $08-3782-1 b(40)$ & $07-1573(40)$ & $07-4556(36)$ & $07-1858(56)$ & $10931(75)$ \\
\hline 4 & $08-7275-1 a(43)$ & $07-1888$ (46) & $07-4751(37)$ & $07-1854(41)$ & $6956(59)$ \\
\hline 5 & $08-3665-1 b(68)$ & 07-1899 (45) & $07-4926(38)$ & $07-2346(43)$ & $10919(82)$ \\
\hline 6 & $08-5386-1 \mathrm{a}(44)$ & 07-1857 (42) & $07-5660(39)$ & 07-2914 (53) & $8026(71)$ \\
\hline 7 & $08-3513-1 \mathrm{a}(42)$ & $07-1855(43)$ & $07-5881(41)$ & $07-8302(25)$ & $5739(38)$ \\
\hline 8 & $08-3513-1 b(\mathrm{NA})^{\mathrm{d}}$ & $07-2687(22)$ & $07-5908(41)$ & $07-9619(38)$ & $6851(56)$ \\
\hline 9 & 08-3488-1b (NA) & $07-2888(25)$ & $07-5929(22)$ & $07-10051(72)$ & $4321(81)$ \\
\hline 10 & 08-5889-1a (45) & $07-3349(23)$ & $07-6000(27)$ & $07-10432(65)$ & $5822(46)$ \\
\hline 11 & & $07-3596(50)$ & $07-6473(48)$ & $09-153(41)$ & $09-240(65)$ \\
\hline 12 & & $07-3651(23)$ & $07-6561(32)$ & $09-640(35)$ & $09-576(65)$ \\
\hline 13 & & $07-5594(44)$ & $07-6858(32)$ & $09-796(57)$ & $09-1183$ (46) \\
\hline 14 & & $07-6334(36)$ & $07-6859(32)$ & $09-875(40)$ & 09-1388 (41) \\
\hline 15 & & $07-6474(32)$ & $07-7288(36)$ & 09-1877 (48) & $09-1645$ (48) \\
\hline 16 & & $07-6439(36)$ & $07-7768$ (27) & 09-2986 (29) & $09-2740(49)$ \\
\hline 17 & & $07-6644(44)$ & $07-8794(47)$ & $09-3072(27)$ & $09-2943$ (68) \\
\hline 18 & & $07-6665(23)$ & 07-9302 (29) & $09-3431(41)$ & $09-3671(62)$ \\
\hline 19 & & 07-6697 (37) & $07-9671(26)$ & $09-3670(39)$ & $09-3675(60)$ \\
\hline 20 & & 07-7713 (49) & 07-9932 (32) & 09-3613 (39) & $09-4161(40)$ \\
\hline 21 & & 07-8301 (37) & $07-10724(50)$ & & \\
\hline 22 & & $07-8663(26)$ & $07-11282(37)$ & & \\
\hline 23 & & $07-8899(36)$ & $07-12365(28)$ & & \\
\hline 24 & & $07-12017$ (24) & $07-13050(40)$ & & \\
\hline 25 & & $07-12230(38)$ & $07-562(35)$ & & \\
\hline 26 & & $07-12320(29)$ & 07-708 (31) & & \\
\hline 27 & & $07-12412(41)$ & $07-879(21)$ & & \\
\hline 28 & & $07-12620(34)$ & $07-915$ (34) & & \\
\hline 29 & & $07-12766(50)$ & $07-934(41)$ & & \\
\hline 30 & & $07-13172(42)$ & $07-1076(25)$ & & \\
\hline
\end{tabular}

aNumber of cases examined. ${ }^{b}$ Samples were collected from patient in different histological type of cervical cancer (different tumor grade or clinical stage) in cervical carcinogenesis (Materials and methods). ${ }^{\mathrm{c}}$ Normal tissue samples are from adjacent tumor tissue. ${ }^{\mathrm{d}}$ Not available.

conditions but are present during carcinogenesis. Accordingly, DNA methylation can be used as a useful biomarker for the diagnosis of many cancers (17). A number of different approaches to genome-wide identification of cancer-associated hypermethylated genes have been developed (9). Nevertheless, at the present time only a few cervical cancer specific methylation markers have been identified. Our goal is to identify more effective and sensitive methylation biomarkers. For this purpose, we have initially focused on evaluating the value of $h L H X 6$-HMR for new methylationbased molecular markers.

The hLHX6 gene encodes a human LIM-homeodomain protein involved in embryogenesis, more specifically in mammalian head development (18). One transcript variant has been described for this gene as $h L H X 6 s$, in which its physiological role is still unknown (19). The $h L H X 6$-HMR is $\mathrm{CpG}$ island located between exon $4 \mathrm{a}$ and exon 5 of the hLHX6s gene on chromosome 9q33.2. This region was originally mentioned by Estácio et al as DIME6 (differentially methylated) in the process of finding an HNSCC (head and neck squamous cell carcinoma) specific biomarker (19). They found several differentially methylated fragments in HNSCC by using methylation-sensitive arbitrarily primed PCR (MS/AP-PCR), one of a genome-wide examination of the methylation pattern, in which DIME6 was found to be the most frequently hypermethylated fragment. Their further studies suggested that DIME6 hypermethylation is a good cancer marker in HNSCC (19). Furthermore, our collaborator (Co. genomictree) showed the different expression levels of $h L H X 6$ gene in normal and cancer cells. This fact led us to 
Table II. Oligonucleotide sequences and conditions for PCR analyses.

\begin{tabular}{|c|c|c|c|c|}
\hline Primer name $^{a}$ & $\begin{array}{l}\text { Primer sequence }{ }^{\mathrm{b}} \\
\text { Forward }(\mathrm{F}) \text { or Reverse }(\mathrm{R})\end{array}$ & Conditions $^{\mathrm{c}}$ & $\begin{array}{l}\text { Amplicon } \\
\text { size (bp) }\end{array}$ & $\begin{array}{l}\text { Sources } \\
\text { or refs. }\end{array}$ \\
\hline pRT-hLHX6-F & CGCGGACGTCCTTCACCGCGG & $69^{\circ} \mathrm{C}, 30 \mathrm{sec}, 40$ cycles & 398 & This study \\
\hline pRT-hLHX6-R & CCGGTTGGAGAGCGGCCCATCC & & & \\
\hline pRT-hLHX6s-1-F & СCTCTGGCTTCTTCCCCTAC & $60^{\circ} \mathrm{C}, 30 \mathrm{sec}, 35$ cycles & 316 & (19) \\
\hline pRT-hLHX6s-1-R & ACTCCTCACCAGTGGACAGC & & & \\
\hline pRT-hLHX6s-2-F & GAGTTTCGGCCTCTCGGCTCAATAG & $60^{\circ} \mathrm{C}, 30 \mathrm{sec}, 35$ cycles & 113 & This study \\
\hline pRT-hLHX6s-2-R & TGGTAGGCGTTGCCGCGAGCTCTCC & & & \\
\hline pMSP-UM-hLHX6s-F & AGGGTTTTAGGGGAAGTGTAGAGT & $55^{\circ} \mathrm{C}, 30 \mathrm{sec}, 35$ cycles & 174 & This study \\
\hline pMSP-UM-hLHX6s-R & ACCAACAATTAAAAAATTAAAACAAC & & & \\
\hline pMSP-M-hLHX6s-F & GTTTTAGGGGAAGCGTAGAGC & $55^{\circ} \mathrm{C}, 30 \mathrm{sec}, 35$ cycles & 170 & This study \\
\hline pMSP-M-hLHX6s-R & CСАACAATTAAAAAATTAAAACGAC & & & \\
\hline pMSP-UM-hLHX6-HMR-F & AGGAGGGGGAGTTTAGTTGT & $58^{\circ} \mathrm{C}, 30 \mathrm{sec}, 35$ cycles & 90 & (19) \\
\hline pMSP-UM-hLHX6-HMR-R & AAATTACAACAAAACCAAAATAACA & & & \\
\hline pMSP-M-hLHX6-HMR-F & AGGAGGGGGAGTTTAGTCGC & $58^{\circ} \mathrm{C}, 30 \mathrm{sec}, 35$ cycles & 85 & (19) \\
\hline pMSP-M-hLHX6-HMR-R & ACGACGAAACCGAAATAACG & & & \\
\hline pBSP-M-hLHX6-HMR-F & cgtaagcttGGGGGTTTTTTTAAGTTTGT & $58^{\circ} \mathrm{C}, 30 \mathrm{sec}, 35$ cycles & 255 & This study \\
\hline pBSP-M-hLHX6-HMR-R & ctagaattcTTCTCATACTTCCAATACATAАAC & & & \\
\hline pCOBRA-hLHX6-HMR-F & TTTTTTGGTTTTTGAGGGG & $60^{\circ} \mathrm{C}, 30 \mathrm{sec}, 35$ cycles & 160 & (19) \\
\hline pCOBRA-hLHX6-HMR-R & TACCAAAATCCCCACCTCA & & & \\
\hline pRT-ACTB-F & AGGTCGGAGTCAACGGATTTG & $58^{\circ} \mathrm{C}, 30 \mathrm{sec}, 21$ cycles & 377 & This study \\
\hline pRT-ACTB-R & GTGATGGCATGGACTGTGGT & & & \\
\hline
\end{tabular}

${ }^{\mathrm{a}} \mathrm{F}$, forward primer; R, reverse primer; $\mathrm{M}$, methylated-specific primers; $\mathrm{U}$, unmethylated-specific primers. ${ }^{\mathrm{b}}$ All sequences shown in the $5^{\prime} \rightarrow 3^{\prime}$ direction. ${ }^{\mathrm{c} C}$ Conditions are shown as the order of annealing temperature $\left({ }^{\circ} \mathrm{C}\right)$, elongation time, and number of cycles. ${ }^{\mathrm{d}}$ Restriction enzymes are represented as italic and lower case.

suspect that the $h L H X 6$-HMR including DIME6 region could also be an effective biomarker in the diagnosis of cervical cancer. Here, we introduce $h L H X 6$-HMR as a sensitive methylation-based molecular biomarker with increased sensitivity and specificity for the early diagnosis of cervical cancer. Moreover, it was of special interest to investigate the epigenetic alterations during multistep cervical cancer development and to determine the tumor stage.

\section{Materials and methods}

Cervical cancer cell lines and human tissue samples. Eight cervical cancer cell lines were used for this study. C33A, $\mathrm{CaSki}, \mathrm{HeLa}$, and $\mathrm{SiHa}$ cells were purchased from the American Type Culture Collection (ATCC). The other cell lines, SNU-17, -703, -1160, and -1299 were obtained from the Korean Cell Line Bank (KCLB, Korea). Each cell line was grown in one of the following media: C33A, HeLa, and SiHa cells in DMEM medium (WelGENE Inc., Korea); CaSki, SNU-703, and SNU-1299 cells in RPMI-1640 medium (Gibco-BRL); SNU-17 and SNU-1160 in AR5 medium (KCLB, Korea). All media were supplemented with $10 \%$ fetal bovine serum (Gibco-BRL) and $1 \%$ antibioticantimycotic (Gibco-BRL). All of these cells were cultured at $37^{\circ} \mathrm{C}$ in a humidified atmosphere composed of $95 \%$ air and $5 \% \mathrm{CO}_{2}$. A total of 110 human tissue samples were kindly provided by Dr Chang-Jin Kim at the Soonchunhyang University Hospital (Cheonan, Korea). These tissue samples originated from cervical cancer patients and their information is represented as the histological tumor grade and age in Table I. These tissue samples for CIN diagnoses were prepared by using microexcision for analysis. Patients provided signed informed consent. The procedure to obtain tissue samples was approved by the Hospital Clinic's Institutional Review Board.

Isolation of total RNA and genomic DNA from cervical cancer cell lines or human tissue samples. Following the manufacturer's instructions, total RNA or genomic DNA was extracted from eight cervical cell lines or human tissue samples using the RNeasy Mini Kit (Qiagen) or DNeasy Blood \& Tissue Kit (Qiagen), respectively.

Reverse transcription ( $R T)-P C R$. For reverse transcription, $1 \mu \mathrm{g}$ RNA of each sample was subjected to cDNA synthesis using oligo (dT) primer and RevertAid First Strand cDNA Synthesis Kit (Fermentas, Korea) according to the manufacture's instruction. PCR amplification was performed using 10 ng cDNA, different sets of primer, and AccuPower PCR PreMix (Bioneer, Korea). The nucleotide sequence of primers and the conditions for gene amplification are shown in Table II. Primers used in this study were synthesized by 


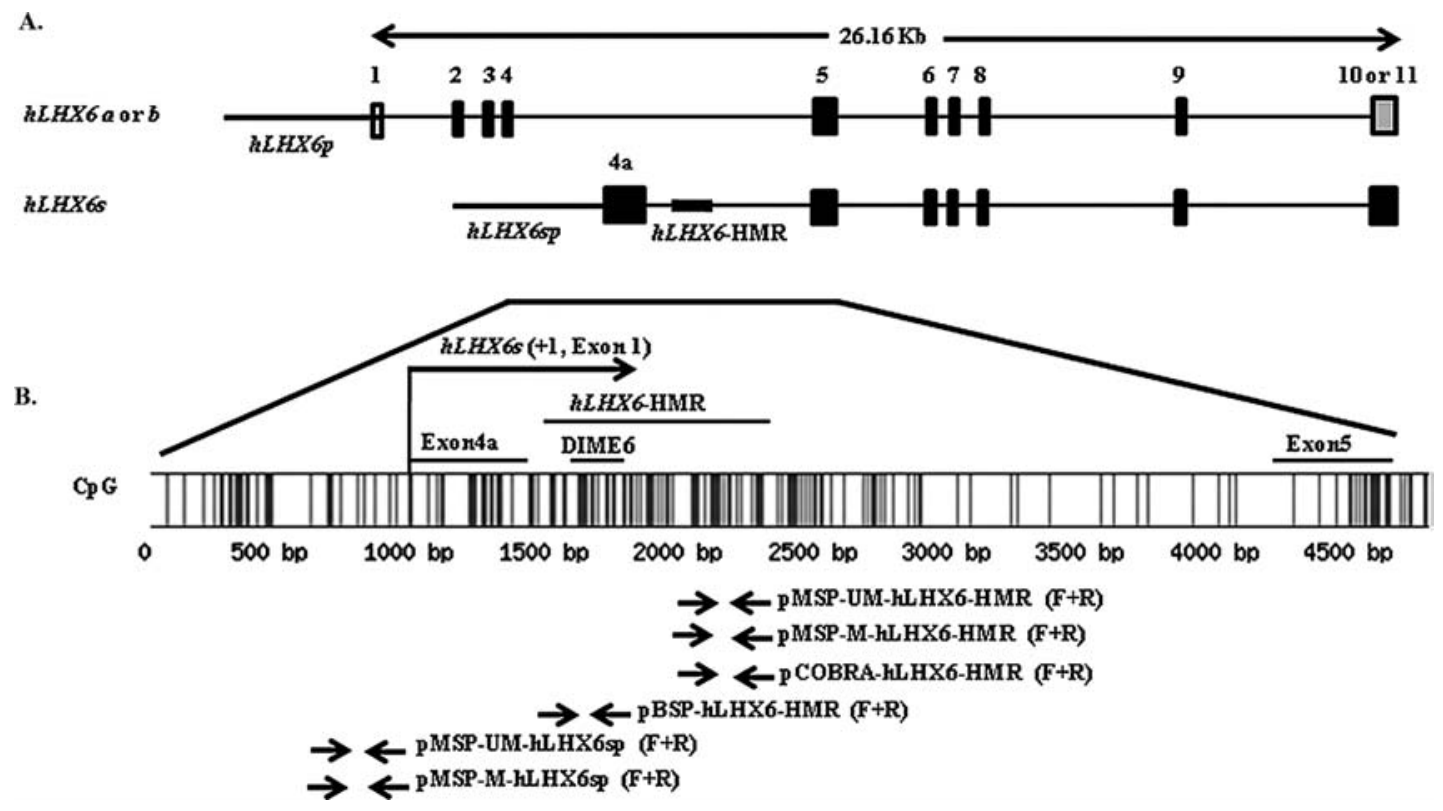

Figure 1. The genomic location and predicted CpG islands of $h L H X 6$-HMR. (A) Genomic structures for $h L H X 6$ and $h L H X 6 s$ genes are represented as exons (black and grey boxes) and introns (thin lines linking the black or grey boxes). Putative minimal promoters of $h L H X 6$ and $h L H X 6 s$ genes, $h L H X 6 p$ and hLHX6sp, are indicated by horizontal thick lines. Numbers indicate the positions of each exon coding $h L H X 6$ and $h L H X 6 s$ genes. The location of $h L H X 6-$ HMR is underlined. (B) CpG dinucleotides in the $h L H X 6 s$ promoter and $h L H X 6$-HMR fragment. CpG sites are shown as vertical lines. Number, +1 , indicates the translation start site of hLHX6s gene. Arrows indicate the position of primers used to amplify each fragment for MSP or BSP assays (Table II). The oligonucleotides used in this study are named as indicated in Fig. 1B. F, forward; R, reverse.

Bioneer (Bioneer, Korea). The 377 bp B-actin gene products were used as an internal control. The amplification reaction was carried out using a GeneAmp PCR System 9700 from Applied Biosystems. The amplification products were electrophoresed on a $2 \%$ agarose gel stained with ethidium bromide and were visualized by using the UV-illuminator or LAS3000 imaging system. The band intensity was measured by using Multi Guage 3.0.

Methylation-specific PCR (MSP). MSP was performed to identify the methylation status in the $h L H X 6 s$ promoter and $h L H X 6$-HMR in human tissue samples as well as in the cervical cancer cell lines. Purified genomic DNA $(1 \mu \mathrm{g})$ was modified using the EZ DNA Methylation Kit (Zymo Research, CA, USA). Bisulfite-treated DNA was then followed by PCR amplification using two pairs of primer for unmethylated and methylated DNAs. The nucleotide sequence of each primer and the amplification conditions are shown in Table II. For all MSP analysis, the PCR mixtures contained 10X reaction buffer, dNTP mixture $(1.0 \mathrm{mM})$, primers (final concentration 10 pmole each per reaction), 1 unit of HotStart prime Taq (Qiagen), and $0.1 \mu \mathrm{g}$ of bisulfitetreated DNA. The methylation status was inferred by the presence or absence of bands and its density was measured by using Multi Gauge 3.0.

Bisulfite sequencing PCR (BSP) analysis. Genomic DNA $(1 \mu \mathrm{g})$ extracted from each cell line or human tissue was bisulfite-treated by using EZ DNA Methylation Kit (Zymo Research). Bisulfite-treated DNA was subjected to PCR reaction using primer pair of pBSP-hLHX6-HMR-F/pBSPhLHX6-HMR-R (Table II). The amplified PCR products were cloned into pBlueScript-SK (+) vector using HindIII and EcoRI restriction enzymes and transformed into DH5 $\alpha$ competent cells. Plasmids purified from amphicillin-positive colonies were sequenced using M13 forward or reverse primer by Solgent (Daejeon, Korea).

Combined bisulfite restriction analysis (COBRA). hLHX6HMR methylation status was also investigated by the COBRA assay (20). One primer set of pCOBRA-hLHX6-HMR-F/ pCOBRA-hLHX6-HMR-R was used to amplify a fragment of $160 \mathrm{bp}$, which can be cleaved into fragments of 31 and 129 bp by digestion with the TaqI restriction enzyme when methylated. Digested PCR products were separated by electrophoresis on $2 \%$ agarose gels and visualized in the LAS-3000 imaging system. Methylation density of $h L H X 6-H M R$ was checked by using Multi Gauge 3.0, in which the density of $129 \mathrm{bp}$ fragment was measured.

Statistical analysis. Statistical analyses were carried out with Statistical Package for the Social Sciences (SPSS) software. Associations of the hLHX6-HMR methylation and cervical carcinogenesis were determined using the $\chi^{2}$ test. Statistical significance was set at $\mathrm{p}<0.05$.

\section{Results and Discussion}

The methylation status of hLHX6-HMR in eight cervical cancer cell lines. The genomic structure of $h L H X 6 s$ promoter and hLHX6s is shown in Fig. 1A, in which hLHX6-HMR is located between exon $4 \mathrm{a}$ and exon 5 of the hLHX6s gene. Nucleotide sequences were obtained from the National Center for Biotechnology Information (http://www.ncbi.nlm.nih. gov/BLAST/). Putative CpG island in $h L H X 6$ s promoter and hLHX6-HMR was predicted using the Methprimer program 
A.

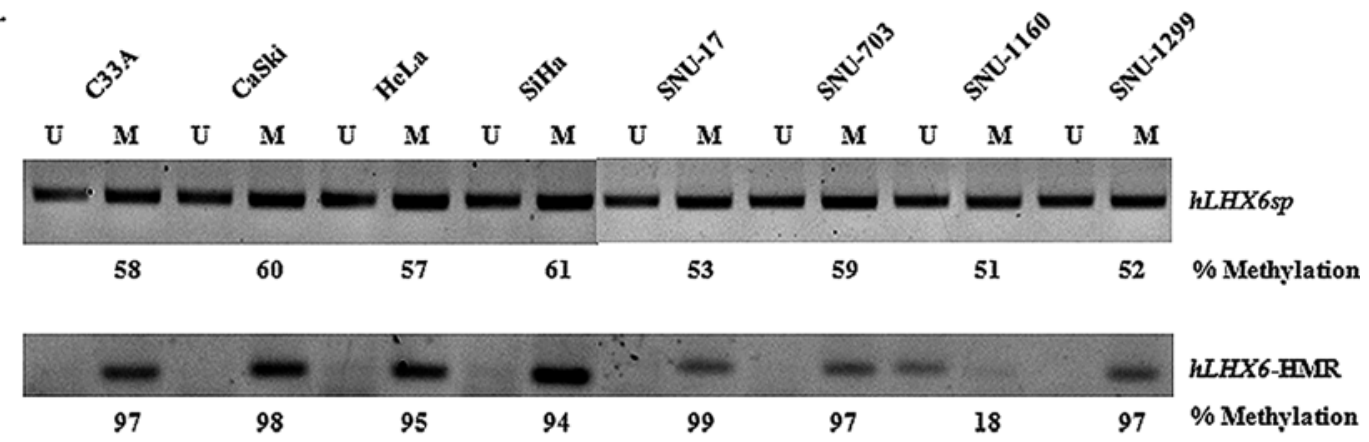

B.

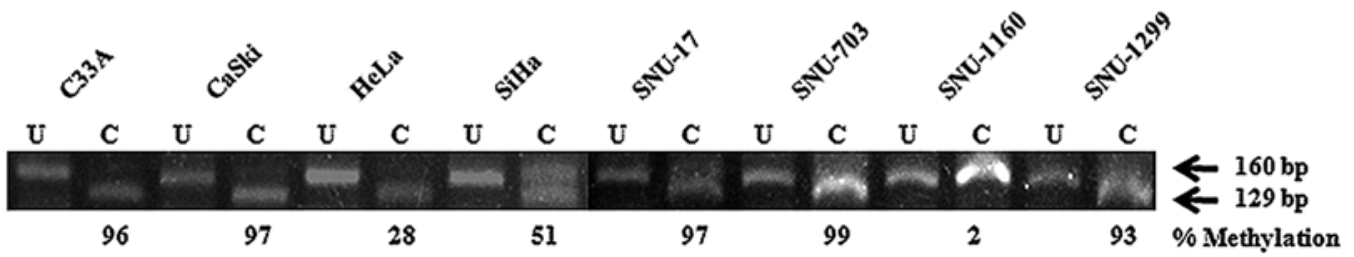

C.

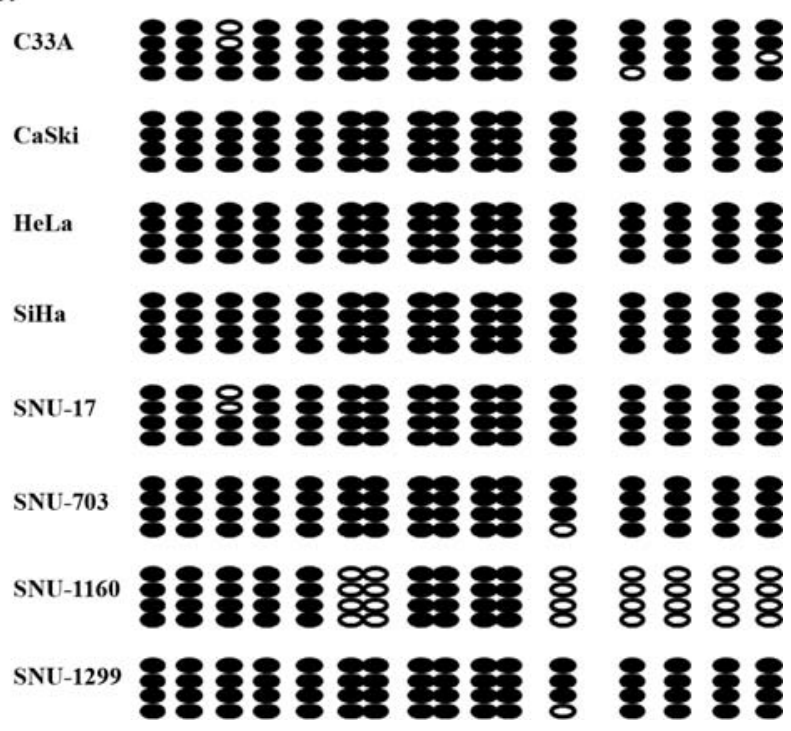

[http://www.urogene.org//methprimer, (21)] with the default setting (\%GC>50\%, ObsCpG/ExpCpG>0.6) (Fig. 1B). CpG dinucleotides in genomic DNA sequences are shown as short vertical lines in Fig. 1B. When compared to the $h L H X 6$ s promoter, a much higher frequency of $\mathrm{CpG}$ dinucleotides is found in $h L H X 6-\mathrm{HMR}$. The methylation levels of these CpG sites were investigated using MSP, BSP, and COBRA assays (Materials and methods). At first, MSP assay was employed to investigate the methylation status in the $h L H X 6$ s promoter and $h L H X 6-H M R$. MSP results showed that only methylation bands were detected in $h L H X 6-\mathrm{HMR}$ of most cell lines except for SUN-1160, in which only unmethylation band was observed (Fig. 2A). Unexpectedly, both unmethylated and methylated PCR products were amplified from the $h L H X 6 s$ promoter with similar densities (Fig. 2A). These results suggested that only $h L H X 6$-HMR was hypermethylated in most cervical cancer cell lines. This conclusion was confirmed by the COBRA assay. In brief, hypermethylation of the
Figure 2. Methylation status of the hLHX6s promoter and $h L H X 6-\mathrm{HMR}$ in 8 cervical cancer cell lines. (A) MSP analysis of the hLHX6s promoter and $h L H X 6 s$-HMR in 8 cervical cancer cell lines. Methylation status is presented by the presence or absence of bands. M represents PCR products amplified by oligonucleotide primers specific for methylated DNA whereas $\mathrm{U}$ indicates PCR products amplified by primers specific for unmethylated DNA. The methylation level is indicated below each sample. (B) Confirmation of the $h L H X 6$-HMR methylation by COBRA assay in 8 cervical cancer cell lines. PCR product was digested by Taq1 restriction enzyme. Abbreviations of $\mathrm{U}$ and $\mathrm{C}$ indicate uncut or cut with TaqI restriction enzyme, respectively. The methylation density is indicated below each sample. (C) Bisulfite sequencing of the $h L H X 6-\mathrm{HMR} \mathrm{CpG}$ sites in 8 cervical cancer cell lines. Methylated or unmethylated cytosines are represented as closed or open circles, respectively. Their spacing reflects the $\mathrm{CpG}$ density of the region. Each row represents an individual cloned allele that was sequenced following sodium bisulfite DNA modification.

hLHX6-HMR was investigated by the digestion of $160 \mathrm{bp}$ DNA fragment with the TaqI restriction enzyme. If they are methylated, amplified 160 bp DNA fragments are cleaved into two fragments of 31 and $129 \mathrm{bp}$ (Materials and methods). Amplified 160 bp PCR fragments from most cell lines were digested by TaqI restriction enzyme (Fig. 2B). PCR fragments amplified from $\mathrm{SiHa}$ cell line were partially digested as shown in Fig. 2B. However, no digested band was found in fragments from SNU-1160 cell line (Fig. 2B). Again, these data suggest that $h L H X 6-\mathrm{HMR}$ is highly methylated in most tested cervical cancer cell lines except for the SNU-1160 cell line. The accuracy of the MSP and COBRA methods in detecting methylated DNA was finally verified by BSP assay. Our data showed that high levels of methylation were exclusively present in most cervical cancer cell lines whereas relatively low levels of methylation were found in the SNU-1160 cell lines (Fig. 2C). When analyzed by MSP, BSP, and COBRA assays, we found concordant results for methylation status of $h L H X 6$-HMR in most tested cell lines. Only one case of SNU-1160 was not consistent in that it seemed to be unmethylated by MSP and COBRA, but not by BSP. Taken together, these results strongly suggest that hLHX6-HMR hypermethylation is a frequent and common event in cervical cancer cell lines. 


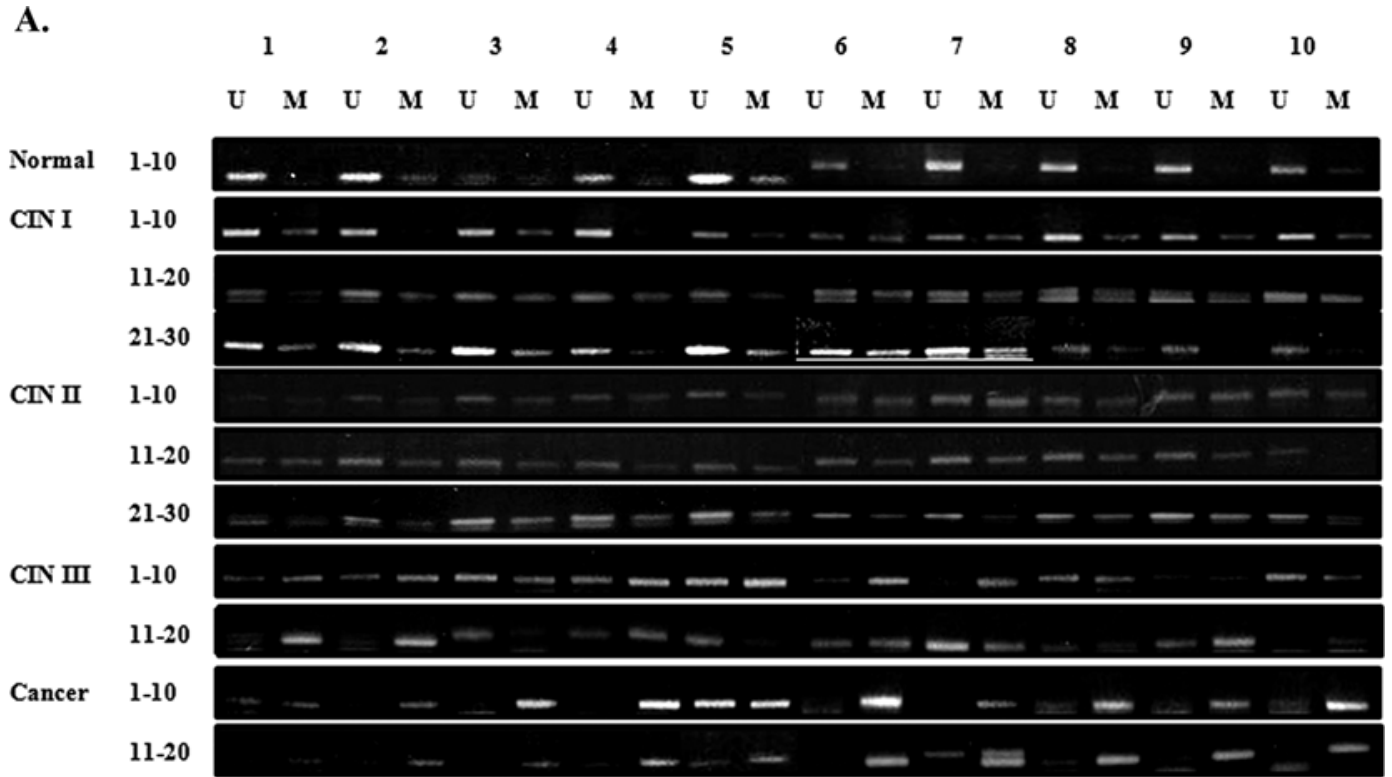

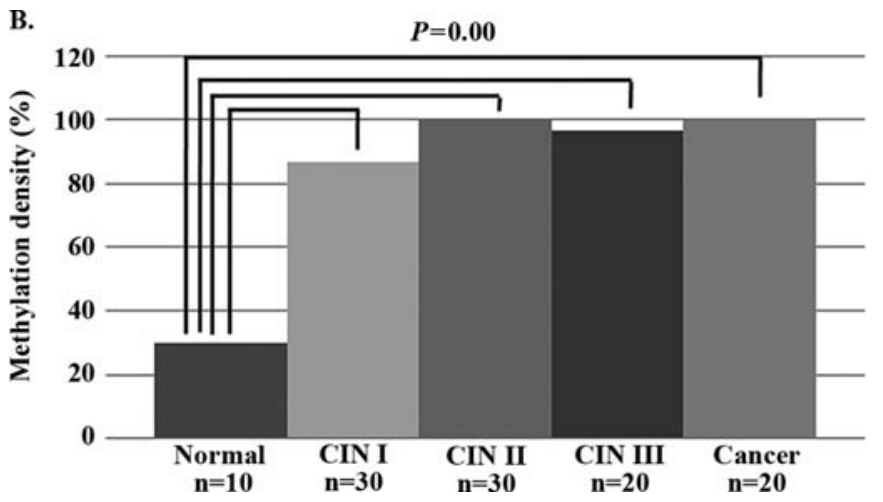

C.

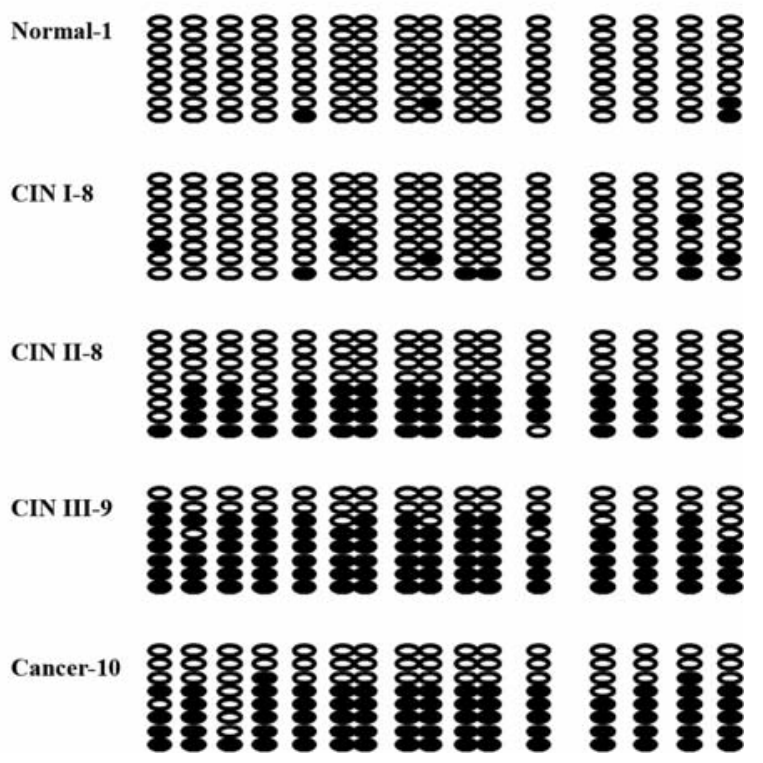

Methylation incidence of hLHX6-HMR during multistep processes of cervical carcinogenesis. Discovery of the correlation between $h L H X 6$-HMR methylation and cervical cancer cell lines suggested the possibility of the same situation in vivo. Therefore, this investigation was extended to human tissue samples from five different stages of cervical carcinogenesis,
Figure 3. Schematic representation of correlation between $h L H X 6-\mathrm{HMR}$ hypermethylation and cervical carcinogenesis. (A) MSP analysis of the hLHX6-HMR in normal, CIN I, CIN II, CIN III, and invasive cervical carcinoma tissues. The symbols of $\mathrm{U}$ or M represent PCR products amplified by oligonucleotide primers specific for unmethylated or methylated DNA, respectively. (B) Bar graph demonstrating the frequency of DNA methylation. Numbers on the $y$-axis are the percentage of positive methylation cases. The $\mathrm{x}$-axis represents the sample types and numbers. The $\mathrm{n}$ indicates the number of tissue samples in each stage and p represents the calculated p-value using the $\chi^{2}$ test for the compared groups. (C) Bisulfite sequencing of the $h L H X 6$-HMR CpG sites in normal, CIN I, CIN II, CIN III, and carcinoma tissue samples. Eight clones were selected from each representative tissue sample and subjected to BSP sequencing. Methylated or unmethylated cytosines are represented as closed or open circles, respectively.

normal cervix, CIN I, CIN II, CIN III, and invasive carcinoma. We evaluated the level of $h L H X 6$-HMR methylation in a total of 110 cervical tissue samples. Genomic DNA extracted from tissue samples was modified by sodium bisulfite and then subjected to MSP and BSP analysis (Materials and methods). MSP analysis was first carried out to examine the methylation density in $h L H X 6$-HMR. Partial or nonmethylated $h L H X 6$-HMR was detected in normal tissue samples, whereas a high degree of methylation was observed in CIN I, CIN II, CIN III, and invasive carcinoma (Fig. 3A). Methylation density in CIN I is clearly higher than that in normal tissues but lower than that in other samples. This result indicates that $h L H X 6$-HMR hypermethylation seems to start from relatively early stages of multistep carcinogenesis. The methylation frequencies of each tissue type are shown in Fig. 3B. The y-axis and $\mathrm{x}$-axis represents the percentage of positive methylation cases and sample types, respectively. Compared to corresponding unmethylated band, a sample was considered hypermethylated when presenting a methylation density superior to $20 \%$. Very low levels of methylation were seen in normal cervix (30\%). The methylation frequencies are very high in CIN I, CIN II, CIN III, and cervical carcinoma tissue samples compared to the normal tissue samples $(\mathrm{p}=0.00)$. It is normally considered that older people have higher levels of methylation compared to younger people. This fact is consistent to our result. When compared the $h L H X 6$-HMR methylation status and the 
A. $1.2 \mathrm{~Kb}$

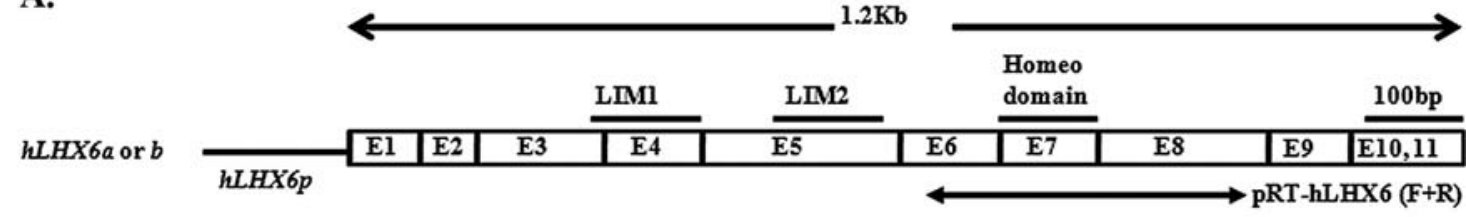

hLHX6s

\begin{tabular}{rl|r|r|r|r|r|r|}
\hline hLHX6sp & E4a & E5 & E6 & E7 & E8 & E9 & E10 \\
\hline & pRT-hLHX6s-1 (F+R) \\
& pRT-hLHX6s-2 (F+R)
\end{tabular}

B.

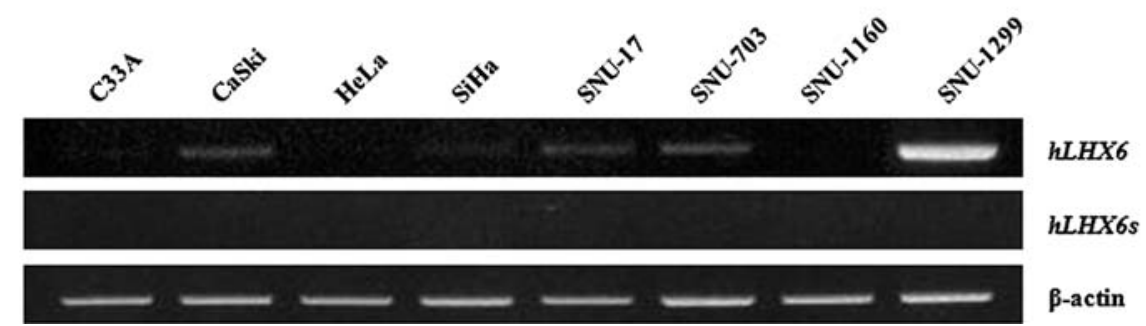

Figure 4. Expression level of $h L H X 6$ and $h L H X 6 s$ genes in 8 cervical cancer cell lines. (A) The full-length mRNA of $h L H X 6$ and $h L H X 6 s$ are shown, in which exons are indicated as boxes and numbers. (B) The transcriptional level of $h L H X 6$ and $h L H X 6 s$ was measured by RT-PCR using different primer pairs as indicated in Materials and methods (Table II). B-actin served as an internal control for the integrity of the cDNA.

ages of the patients group studied, significant association of methylation and age was found, in which older patients showed higher levels of DNA methylation in $h L H X 6$-HMR. BSP was performed to confirm the result of MSP. Eight clones from representative sample were sequenced and evaluated for the frequency of $h L H X 6$-HMR methylation (Materials and methods). None of the normal and CIN I cells exhibited hypermethylation. In contrast, CIN II, CIN III, and invasive carcinoma cells showed high levels of methylation in $h$ LHX6HMR (Fig. 3C). In general, BSP result was consistent with that of MSP except for the case of CIN I.

In conclusion, our analysis evaluating methylation in cervical tissues led to two conclusions. First, $h L H X 6-H M R$ methylation is associated with cervical carcinogenesis. Second, this abnormal DNA methylation occurs at the relatively early stages during tumorigenesis of cervical cancer. This finding suggests a potential clinical application of hLHX6-HMR methylation as an important molecular marker for the early diagnosis of cervical cancer.

Expression of hLHX6 and hLHX6s genes in eight cervical cancer cell lines. Our methylation analysis showed a nearly perfect correlation between the $h L H X 6$-HMR hypermethylation and cervical carcinogenesis. The data implied a negative effect of hypermethylated $h L H X 6$-HMR on $h L H X 6$ or $h L H X 6 s$ gene expression. In fact, Estácio et al suggested that hypermethylation of $h L H X 6-\mathrm{HMR}$ is associated with transcriptional silencing of $h L H X 6 s$ in HNSCC cell lines (19). Therefore, we performed RT-PCR on 8 cervical cancer cell lines to investigate the same effect in cervical cancer cells (Materials and methods). Fig. 4A shows the fulllength transcripts for $h L H X 6$ and $h L H X 6$ s genes. The level of $h L H X 6$ gene expression was found to be relatively low in most cell lines but high in SNU-1299 (Fig. 4B). However, the transcript of the $h L H X 6$ s gene was not detected in any cervical cancer cell lines. Inverse correlation between the methylation level and mRNA quantity was at first believed to be due to the methylation in $h L H X 6$-HMR as was the case in the result of Estácio et al. However, we could not exclude another possibility. It might be just due to the tissue specific gene repression in the cervix not to the hypermethylation in hLHX6-HMR. Therefore, hLHX6s gene expression was tested in normal cervix tissues as well as in cervical cancer tissues. Our results showed that no transcription was detected in either of the tissues (data not shown). In contrast to the data of Estácio et al, our study suggested that the hLHX6HMR methylation is not associated with the transcriptional silencing of the $h L H X 6 s$ gene in the cervix. Instead, it is speculated that the methylation of $h L H X 6$-HMR may have an effect on the epigenetic regulation of other isoforms. Estácio et al suggested that $h L H X 6$ is transcribed in almost all investigated tissues but that hLHX6s was expressed only in brain, colon, small intestine and testis of eleven other normal tissues (19). Therefore, we concluded that $h L H X 6 s$ gene expression may be tissue specific.

Taken together, these results indicate that $h L H X 6$-HMR can be used as an effective and sensitive biomarker for early detection of cervical cancer. Moreover, this biomarker seems to have increased sensitivity and specificity for cervical cancer. In addition, better understanding of the pattern of the methylation event in this gene may lead to the determination of its biological function. Therefore, our study may contribute to the prevention and optimal treatment of cervical cancer by early detection.

\section{Acknowledgements}

This study was supported by the Research Center for Women's Diseases, at the Korea Science and Engineering Foundation and Sookmyung Women's University (2008). 


\section{References}

1. Pisani P, Parkin DM, Bray F and Ferlay J: Erratum: estimates of the worldwide mortality from 25 cancers in 1990 . Int J Cancer 83, 18-29 (1999). Int J Cancer 83: 870-873, 1999.

2. Durst M, Glitz D, Schneider A and Zur HH: Human papillomavirus type 16 (HPV 16) gene expression and DNA replication in cervical neoplasia: analysis by in situ hybridization. Virology 189: 132-140, 1992

3. Bosch FX, Manos MM, Munoz N, Sherman M, Jansen AM, Peto J, Schiffman MH, Moreno V, Kurman R and Shah KV: Prevalence of human papillomavirus in cervical cancer: a worldwide perspective. International biological study on cervical cancer (IBSCC) Study Group. J Natl Cancer Inst 87: 796-802, 1995.

4. Zur HH: Papillomaviruses and cancer: from basic studies to clinical application. Nat Rev Cancer 2: 342-350, 2002.

5. Walboomers JM, Jacobs MV, Manos MM, Bosch FX, Kummer JA, Shah KV, Snijders PJ, Peto J, Meijer CJ and Munoz N: Human papillomavirus is a necessary cause of invasive cervical cancer worldwide. J Pathol 189: 12-19, 1999.

6. The 1988 Bethesda System for reporting cervical/vaginal cytological diagnoses: National Cancer Institute Workshop. JAMA 262: 931-934, 1989

7. Agorastos T, Miliaras D, Lambropoulos AF, Chrisafi S, Kotsis A, Manthos A and Bontis J: Detection and typing of human papillomavirus DNA in uterine cervices with coexistent grade I and grade III intraepithelial neoplasia: biologic progression or independent lesions? Eur J Obstet Gynecol Reprod Biol 121: 99-103, 2005.

8. Asakawa S, Tsunematsu K, Takayanagi A, Sasaki T, Shimizu A, Shintani A, Kawasaki K, Mungall AJ, Beck S, Minoshima S and Shimizu N: The genomic structure and promoter region of the human parkin gene. Biochem Biophys Res Commun 286: 863-868, 2001.
9. Laird PW: The power and the promise of DNA methylation markers. Nat Rev Cancer 3: 253-266, 2003.

10. Baylin SB and Ohm JE: Epigenetic gene silencing in cancer - a mechanism for early oncogenic pathway addiction? Nat Rev Cancer 6: 107-116, 2006.

11. Jones PA and Baylin SB: The fundamental role of epigenetic events in cancer. Nat Rev Genet 3: 415-428, 2002.

12. Costello JF, Fruhwald MC, Smiraglia DJ, Rush LJ, Robertson GP, Gao X, Wright FA, Feramisco JD, Peltomaki P, Lang JC, Schuller DE and Yu L: Aberrant CpG-island methylation has non-random and tumour-type-specific patterns. Nat Genet 24: 132-138, 2000.

13. Esteller M, Corn PG, Baylin SB and Herman JG: A gene hypermethylation profile of human cancer. Cancer Res 61: 3225-3229, 2001.

14. Issa JP: $\mathrm{CpG}$ island methylator phenotype in cancer. Nat Rev Cancer 4: 988-993, 2004.

15. Ushijima T: Detection and interpretation of altered methylation patterns in cancer cells. Nat Rev Cancer 5: 223-231, 2005.

16. Egger G, Liang G, Aparicio A and Jones PA: Epigenetics in human disease and prospects for epigenetic therapy. Nature 429: 457-463, 2004.

17. Jones PA and Buckley JD: The role of DNA methylation in cancer. Adv Cancer Res 54: 1-23, 1990.

18. Grigoriou M, Tucker AS, Sharpe PT and Pachnis V: Expression and regulation of Lhx6 and Lhx7, a novel subfamily of LIM homeodomain encoding genes, suggests a role in mammalian head development. Development 125: 2063-2074, 1998.

19.Estácio MR, Youssef EM, Rahal P, Fukuyama EE, Gois-Filho JF, Maniglia JV, Goloni-Bertollo EM, Issa JP and Tajara EH: LHX6 is a sensitive methylation marker in head and neck carcinomas. Oncogene 25: 5018-5026, 2006.

20. Xiong $\mathrm{Z}$ and Laird PW: COBRA: a sensitive and quantitative DNA methylation assay. Nucleic Acids Res 25: 2532-2534, 1997.

21. Li LC and Dahiya R: MethPrimer: designing primers for methylation PCRs. Bioinformatics 18: 1427-1431, 2002. 